\title{
E. coli Bacteremia Strains - High diversity and Associations with Age- related Clinical Phenomena
}

\section{Wester $\mathrm{AL}^{1 *}$, Melby $\mathrm{KK}^{2,3}$, Wyller $\mathrm{TB}^{2,4}$ and Dahle UR ${ }^{1}$}

${ }^{1}$ Division of Infectious Disease Control, Norwegian Institute of Public Health, Norway

2Institute of Clinical Medicine, University of Oslo, Norway

${ }^{3}$ Department of Microbiology, Oslo University Hospital, Norway

${ }^{4}$ Department of Geriatric medicine, Oslo University Hospital, Norway

\begin{abstract}
Advanced age is associated with an increased proportion of Escherichia coli in bacteremia as well as an increased risk of death from $E$. coli blood stream infection. Age-associated differences in normal flora $E$. coli indicate that elderly persons may be diseased by other groups of $E$. coli than do younger patients. We studied a historical cohort of 212 patients with community-acquired $E$. coli bacteremia. The bacterial strains were tested for antimicrobial resistance and analyzed by a generic Multi-Locus Variable-Tandem Repeats Analysis (MLVA).

The available 212 strains showed a great diversity, and clustered into ten different MLVA-type complexes (MTC) MTC-b, containing 97 of the strains, was associated with $\geq 1$ comorbid illness (OR 2.02, 95\% Cl 1.12-3.64), and with to $\geq 1$ atypical symptom (OR $0.46,95 \% \mathrm{Cl} 0.27-0.80)$. MTC-c, containing 31 strains, was associated with urinary origin of infection (OR $3.28,95 \% \mathrm{Cl} 1.345-8.00)$ and was preventive against gastrointestinal origin of infection (OR $0.11,95 \% \mathrm{Cl} 0.01-0.83)$. MTC-g, containing only eight strains, was associated with leukopenia (OR $6.43,95 \% \mathrm{Cl}$ 1.15-36.00). The strains showed low level of antimicrobial resistance. Fifteen of the 212 patients (7.1\%) died within 14 days after admission to hospital. Neither MTC nor antimicrobial resistance was associated with hospital mortality.

In conclusion, our study showed a great degree of diversity of the strains and that one of the MTCs was associated with age-related clinical phenomena. None of the MTCs were associated with outcome, indicating that patient characteristics are more important than microbial characteristics.
\end{abstract}

Keywords: E. coli, age, bactermia,genotype, mortality

\section{Introduction}

E. coli is the most frequent Gram-negative bacillus isolated in blood stream infections [1,2]. Unlike other common pathogens seen in community-acquired blood stream infections, the proportion of $E$. coli isolates increases with increasing age [1]. Clinical as well as population-based studies have identified high age, in addition to certain clinical factors such as hospital acquisition, comorbid illnesses, presence of shock, non-urinary focus, and antimicrobial resistance in conjunction with inadequate treatment, as risk factors for death in E. coli blood stream infection $[3,4]$. Unlike commensal and intestinal pathogenic E. coli, extra intestinal pathogenic E. coli (ExPEC) derive predominantly from E. coli main phylogenetic group B2, and to a lesser extent, group D [5]. In a mouse model, phylogeny was linked to virulence [6]. However, clinical studies of E. coli bacteremia give conflicting results when comparing relative impact of host and bacterial determinants on severity of infection [7-9]. Advanced age is associated with an increased risk of severe infection, probably due to immune dysregulation and a more pronounced procoagulant state [10] as well as increased apoptosis [11]. The above-mentioned studies did not, however, evaluate whether advanced age and age-related clinical presentation were associated with different types of $E$. coli. Efforts are made to establish strategies other than antibiotic treatment to manage or prevent ExPEC infections, based on certain virulence traits [12], or by using more of a whole-cell approach [13]. It is therefore relevant to ask whether elderly patients fall seriously ill from other groups of $E$. coli than do younger patients.

A fast and easy molecular tool to discriminate different isolates of the same species is Multiple-Locus Variable number tandem repeats Analysis (MLVA) [14]. The National Reference Laboratory for Enteropathogenic Bacteria at the Norwegian Institute of Public Health routinely uses a generic MLVA protocol to genotype enteropathogenic E. coli of all serotypes [15]. By applying a 9-locus variant of the recently updated version [16] of the original protocol [17], the aim of the study was to determine if high age and age-linked clinical phenomena were associated with certain genotypes of $E$. coli found in bacteremia cases already well described [18]. Furthermore, we aimed to determine whether particular bacterial genotypes or antimicrobial resistance represented risk factors for hospital mortality.

\section{Materials and Methods}

\section{Patients and $E$. coli isolates}

Demographic, laboratory and clinical data for 450 adult patients with $E$. coli bacteremia admitted to a middle-sized Norwegian hospital between 1994 and 2004 was part of a clinical-epidemiological study described elsewhere [18]. A total of $225 \mathrm{E}$. coli strains isolated from patients admitted between 2000 and 2004 could be retrieved from the -70 degree centigrade freezer at Aker University Hospital. Of these, 212 isolates were verified as $E$. coli by matrix-assisted laser desorption ionization-time of flight mass spectrometry (MALDI-TOF) technology,

*Corresponding author: Astrid Louise Wester, Division for Infectious Disease Control, Norwegian institute of public health, P.O. Box 4404 Nydalen, N-0403 Oslo, Norway, Tel: +47 21077000; Fax +47 22353605; E-mail: astrid.louise.wester@fhi.no

Received January 20, 2014; Accepted February 17, 2014; Published February 25, 2014

Citation: Wester AL, Melby KK, Wyller TB, Dahle UR (2014) E. coli Bacteremia Strains - High diversity and Associations with Age-related Clinical Phenomena. Clin Microbial 3: 140. doi:10.4172/2327-5073.1000140

Copyright: (c) 2014 Wester AL, et al. This is an open-access article distributed under the terms of the Creative Commons Attribution License, which permits unrestricted use, distribution, and reproduction in any medium, provided the original author and source are credited. 
whereas five were non-E. coli (one Citrobacter, three Klebsiella, one Morganella morganii and one not further specified) and a further five did not grow after thawing. As a result, 212 isolates and respective patients (median age 78 years, interquartile range 61-83 years) were eligible for the study.

As markers of microbial phylogeny in the molecular statistical analyses, we included 42 human strains from the E. coli Reference (ECOR)-collection obtained from the Microbial Evolutionary Laboratory, Michigan, State University, East Lansing, USA, as well as four shiga toxin producing E. coli (STEC) strains associated with Haemorrhagic Uremic Syndrome (HUS) from the strain collection at the Norwegian Institute of Public Health. In the analyses we also included the recently described results on the presence of the shiga toxin gene st $x 2$ for 193 of the strains [19].

\section{Microbiological analyses}

Testing of antimicrobial susceptibility: All strains confirmed to be E. coli were tested for antimicrobial susceptibility by the EUCAST standardized disc-diffusion method version 3.0 (http://www.eucast.org/ antimicrobial_susceptibility_testing/disk_diffusion_methodology/). The panel of antimicrobials tested was in accordance with the panel recommended by the Norwegian Working Group on Antimicrobials (http://www.unn.no/resistenspaneler/category19025.html). Based on millimetre zones, antimicrobial susceptibility was categorized into sensitive, intermediately sensitive and resistant according to EUCAST clinical breakpoints version 3.0 (http://www.eucast.org/ clinical_breakpoints/). The strains were screened for resistance against third generation cephalosporins by cefpodoxime, and, if reduced susceptibility was found, phenotypical and molecular analyses were performed to detect Extended Spectrum Betalactamase (ESBL) activity. Resistance to one or both of the antimicrobials used as empirical treatment during the study period (ampicillin and gentamicin combined for severe infections presumably located below the diaphragm) was categorized as ampicillin-gentamicin resistant (AG-resistant), whereas multidrug resistance (MDR) and extensive drug-resistance (XDR) were defined according to the 2011 joint ECDC/CDC definitions [20].

\section{MLVA analysis}

Genomic DNA of E. coli was prepared by 15 minutes of boiling of strain suspensions and used directly in the PCR reaction after centrifugation at $960 \mathrm{~g}$ for three minutes. The isolates were MLVA genotyped in accordance with the previously described protocols $[16,17]$. The following loci were included: CVN001, CVN002, CVN003, CVN004, CVN007, CVN014, CVN015, CVN016, and CVN017. Repeats were amplified using Qiagen Multiplex PCR-kit (Qiagen, Hilden, Germany).

PCR products were subjected to capillary electrophoresis on an ABI 3130 Genetic Analyser (Applied Biosystems, Foster City, CA, USA). Each peak was identified according to colour and size, and allele numbers were assigned based on fragment sizes. Alleles lacking amplicons were designated as having zero alleles.

\section{Statistical analyses}

The rate of patients lost to follow-up (i.e. from the initially available 450 isolates) was $52.9 \%$. Chi-squared tests were used for evaluating if there were statistically significant differences in clinical and demographic variables between patients included and those lost.

Clinical and demographic variables have previously been found to be independently associated with hospital death within 14 days of admission [18]. These variables were selected for statistical analysis in the current study. For analytical purposes we dichotomized age, number of comorbid illnesses, leukopenia and fever at the same cut-off values as previously described [18]. Chi-squared tests and Fisher's exact tests were used.

The allele numbers generated from the MLVA-analyses were entered into BioNumerics (Version 6.6, Applied Maths, SintMartens-Latem, Belgium) as character values and a dendrogram was constructed using categorical coefficients and the UPGMA algorithm. Numerical values were assigned for distinct MLVA type profiles, and MLVA-Type Complexes (MTCs) were assigned for related isolates with similarity $>57 \%$. Mantel-Haenzel Common Odds Ratio estimation was used to analyse whether different MTCs were associated with age, gender and different clinical variables, as well as with the presence of stx2. Chi-squared tests were used to test whether microbial and clinical variables were associated with death within 14 days after admission to hospital. In order to explore possible grouping of clinical outcome and stx2-status to phylogenetic groups of ECOR strains or to HUSassociated STEC, an analysis based on Minimal Spanning Tree (MST) hierarchical clustering for categorical values was performed.

\section{Results}

There were no systematic differences with regard to the selected variables between patients included and patients from whom no strain was available. Of the included patients, $147(69.3 \%)$ patients were 65 years or older. A total of 133 patients (62.7\%) were female. Among the

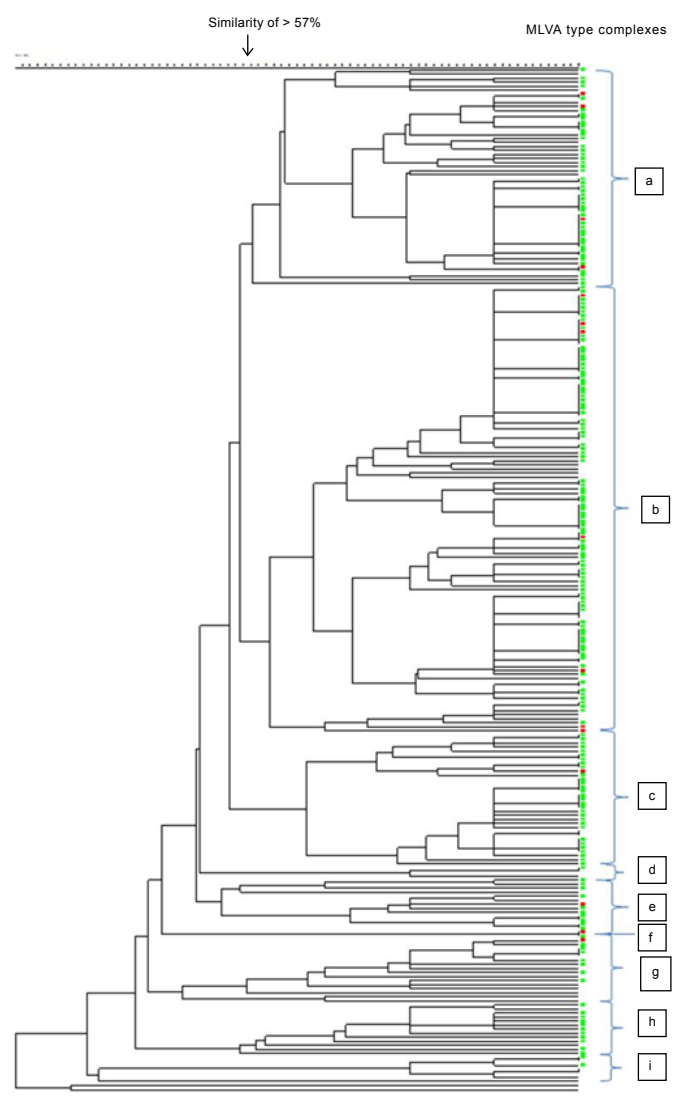

Figure 1: Dendrogram of the MLVA results of 212 bacteremia isolates of $E$. coli, 42 ECOR strains of human origin and 4 HUS-associated STEC strains. Blood culture isolates found in patients that died and were alive 14 days after admission to hospital in red and green, respectively. 
Citation: Wester AL, Melby KK, Wyller TB, Dahle UR (2014) E. coli Bacteremia Strains - High diversity and Associations with Age-related Clinical Phenomena. Clin Microbial 3: 140. doi:10.4172/2327-5073.1000140

patients, $55.6 \%$ had no organ failure within one day of admission to hospital, whereas $28.3 \%, 10.4 \%$ and $4.7 \%$ had one, two and $\geq 3$ failing organs within one day of admittance, respectively. The MTCs were grouped into MTC a-i according to the MLVA clustering shown in Figure 1 and in Annexure 1. Figure 3 shows odds ratio (OR)-plots for the associations between MTCs and demographic and clinical variables. The largest MTC, containing 97 of the strains, was MTC-b. This MTC was associated with $\geq 1$ comorbid illness (OR 2.02, 95\% CI 1.12-3.64), and acted to prevent having $\geq 1$ atypical symptom (OR $0.46,95 \%$ CI 0.27-0.80). MTC-c, containing 31 strains, was associated with urinary site of infection (OR 3.28, 95\% CI 1.35-8.00) and indicated reduced probability of gastrointestinal origin of infection (OR 0.11, 95\% CI
0.01-0.83). MTC-g, containing only eight strains, was associated with leukopenia (OR 6.43, 95\% CI 1.15-36.00). None of the MTCs were associated with early organ failure, neither when assessed as having $\geq 1$ failing organ, nor when assessed as having $\geq 3$ failing organs (results not shown), within one day of admission.

Fifteen of the 212 patients ( $7.1 \%$ ) died within 14 days after admission to hospital. The results regarding age, comorbidity, clinical variables and microbial characteristics as risk factors for hospital mortality are presented in Table 1. Antimicrobial resistance was not associated with hospital mortality within 14 days after admission, and none of the MTCs were associated with severity of infection, neither when assessed as assessed as having $\geq 3$ failing organs within one day of admission

\begin{tabular}{|c|c|c|c|c|}
\hline & $\begin{array}{l}\text { Total Material (212 Patients; } \\
\text { \%within groups) }\end{array}$ & $\begin{array}{l}\text { Alive at } 14 \text { days ( } 188 \text { Patients; } \\
\% \text { within this group) }\end{array}$ & $\begin{array}{l}\text { Patients that died within } 14 \text { days } \\
\text { (24 patients; \% within this group) }\end{array}$ & P-Value* \\
\hline Age $\geq 65$ Years & $148(69.8)$ & $134(68.0)$ & $14(93.3)$ & 0.042 \\
\hline Cornorbid illness $\geq 1^{1}$ & $139(65.9)$ & $124(63.3)$ & $15(100)$ & 0.003 \\
\hline Fever $\left(\geq 38.5^{\circ} \mathrm{C}\right)$ & $146(69.5)$ & $138(70.8)$ & $8(53.3)$ & 0.16 \\
\hline Leukopenia $(<3000 / \mu \mathrm{L})$ & $12(5.7)$ & $5(2.6)$ & $7(46.7)$ & $<0.001$ \\
\hline Early Organ Failure ${ }^{2}$ & $92(44.4)$ & $78(40.4)$ & $144(100)$ & $<0.001$ \\
\hline \multicolumn{5}{|l|}{ Antimicrobial resistance } \\
\hline -against $3^{\text {rd }}$ generation Cepalosporins & $3(1.4)$ & $2(1.0)$ & $1(6.7)$ & 0.20 \\
\hline $\begin{array}{l}\text {-against agents used as empicial treatment } \\
\text { (ampicillin or gentarricin) }\end{array}$ & $1(0.5)$ & $1(0.5)$ & 0 & 1.00 \\
\hline Multiresistance (against $\geq 3$ groups of agents tested) & $48(22.7)$ & $46(23.6)$ & $2(13.3)$ & 0.53 \\
\hline \multicolumn{5}{|c|}{ MLVA-type complex (MTC only those containing at least five strains) } \\
\hline MTC-a & $49(23.1)$ & $45(22.8)$ & $4(26.7)$ & 0.735 \\
\hline MTC-b & $97(45.8)$ & $90(45.7)$ & $7(46.7)$ & 0.941 \\
\hline MTC-c & $31(14.6)$ & $30(15.2)$ & $1(6.7)$ & 0.366 \\
\hline MTC-e & $11(5.2)$ & $10(5.1)$ & $1(6.7)$ & 0.789 \\
\hline MTC-g & $8(3.8)$ & $7(3.6)$ & $1(6.7)$ & 0.542 \\
\hline MTC-g & $11(5.2)$ & $11(5.6)$ & 0 & 0.347 \\
\hline
\end{tabular}

${ }^{*}$ Chi-Square test or Fishers test. ${ }^{1}$ Cornorbid illness: Malignant Disease, Chronic Atrial Fibrillation, Ischemic heart Disease, Hypertension, Heart disease, Cerebrovascula disorder, Chronic obstructive lung disease, Alcoholism, Diabetes diseases, Chronic renal Failure, ${ }^{2}$ Presence of $\geq 1$ failing organ within one day after admission.

Table 1: Risk factors for hospital death within 14 days after admission.
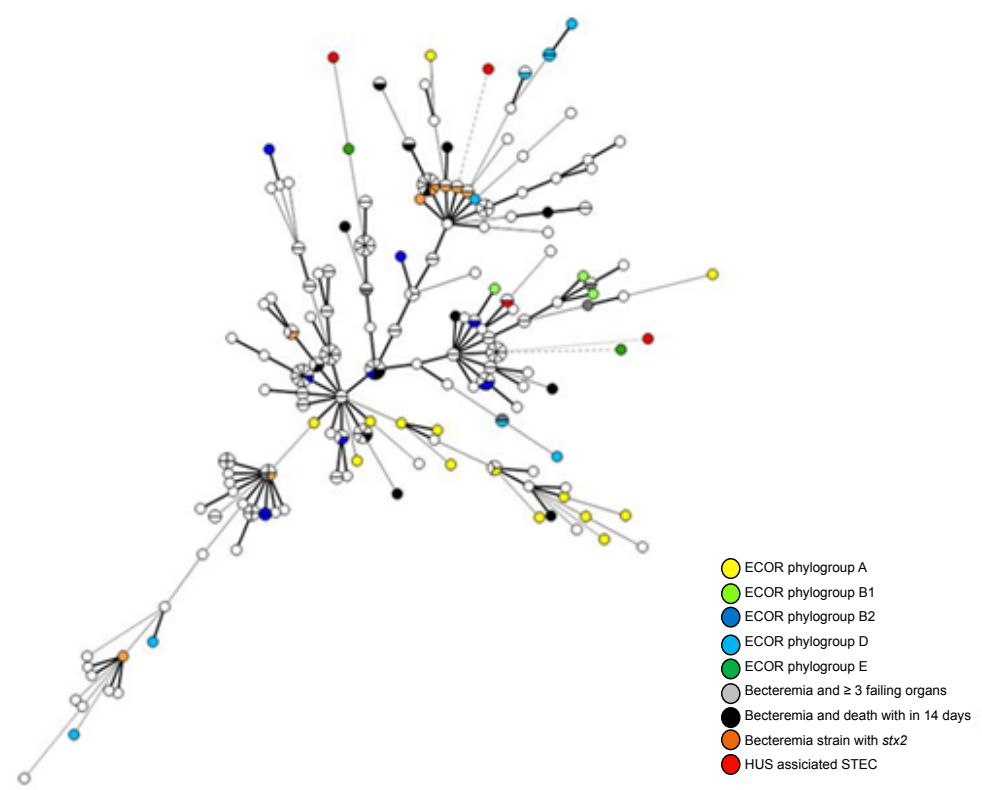

Figure 2 (a): Minimum spanning tree of 212 bacteremia isolates of $E$. coli (outcome in white, grey or black), 42 ECOR strains of human origin (with known phylogroup in colours) and 4 HUS-associated STEC (in red colour). 


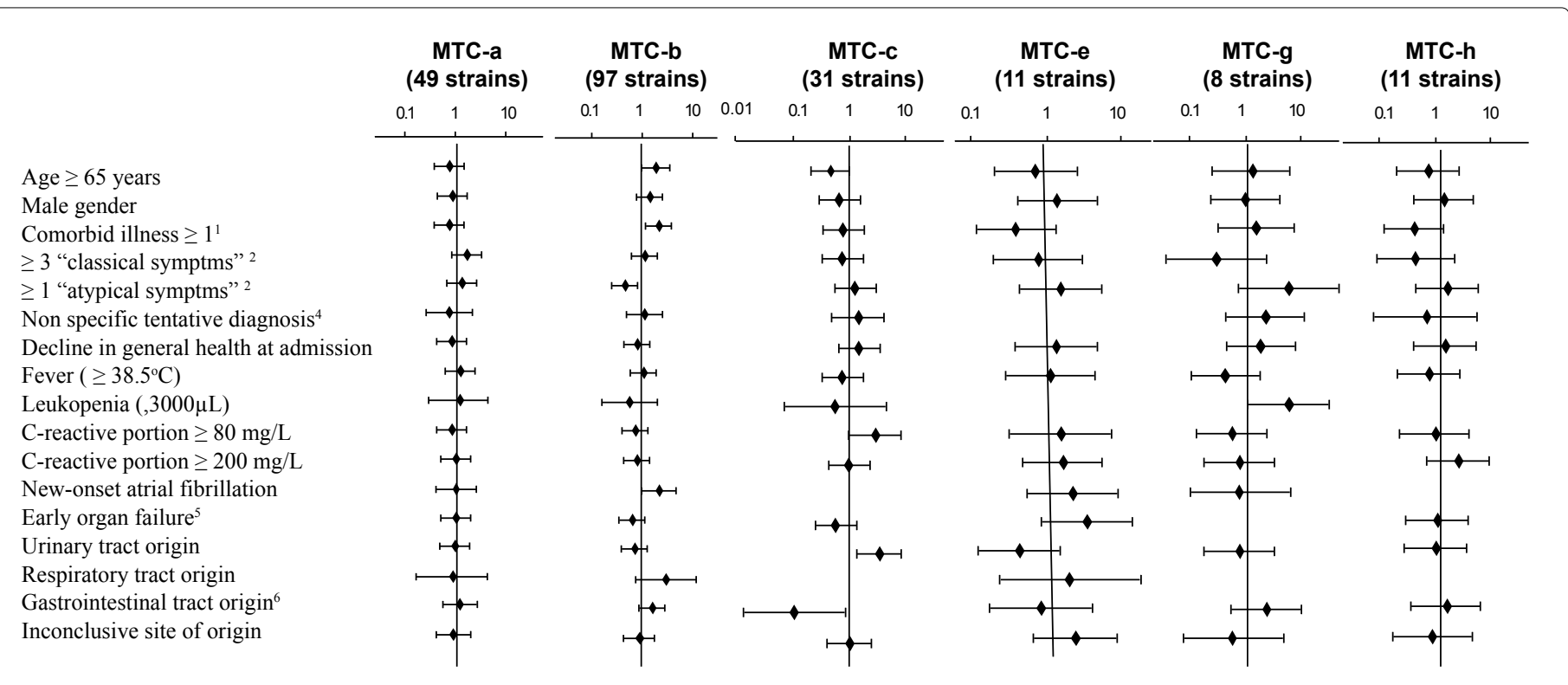

*Only MfCs with $\geq 5$ bacteremia strains included

${ }^{1}$ Comorbid illnesses: Malignant disease,chronic atrial fibrillation, ischemic heart disease,hypertension, heart failure,cerebrovascular disorder, chronic obstructive lung disease, alcoholism, diabetes mellitus, chronic renal failure

2"Classical symptoms": fever/chills, localised pain, nausea/vomiting, diarrhea, cough, dyspnea, expectoration, urgency, painful voiding, hematuria, skin rash and coma/seizures 3"Atypical symptoms": Malaise, falls, dizziness, syncope, unsteadiness, immobility, acute incontinence of urine or feces, paresis/speaking difficulties,

acute confusional state

${ }^{4}$ Non-specific tentative diagnoses: acutedeterioration of performing daily tasks, re1uced general condition, confusion, dizziness, falls, fainting, question of cerebral infarction ${ }^{5}$ Presence of 1 failing organ within one day after admission

${ }^{6}$ Gastrointestinal tract including liver, pancreas and biliary tract

Figure 3: Odds ratio OR plots with $95 \%$ confedence intervals of associations between clinical presentation and MLVA complex (MTC) ${ }^{\star}$.

(results not shown), nor with mortality within 14 days of admission to hospital. The latter is also illustrated in the MST in Figure 2a.

Figures 1 and 2 show a high degree of diversity among the bacteremia E. coli strains included. Neither strains isolated from patients with severe infection, nor the HUS-associated STEC strains, seemed to be located in any specific branch of the tree. As expected, ECOR strains of all main phylogroups did not cluster, except for ECOR strains belonging to main phylogroup A. As shown in Figures 2a and $2 \mathrm{~b}$ the latter (Included as supplementary data), these strains grouped together with eleven bacteremia isolates (5.2\%) belonging mostly to MTC-g (8 of 11 strains). Three of these originated from urinary tract infection, out of a total of 116 strains (2.6\%) with urinary tract origin. One strain was isolated from a patient who died within 14 days of hospital admission.

Additionally, Figure 2a shows that six of the nine stx2-positive bacteremia isolates were grouped together with each other, and together with the HUS-associated O103 STEC strain, as well as with ECOR strains of different phylogroups (one phylogroup A strain, five phylogroup B1 strains, and one B2 strain). Most bacteremia strains in this main branch of the MST belonged to MTC-a (Figure 2b Included as supplementary data), and the association between stx2-status and MTC-a was formally confirmed (p-value 0.002). Five of the six stx2positive isolates were subtype stx2c, and all patients in question had been admitted to hospital during the winter/spring of 2002. Also, the two other stx2c-positive isolates, which, however, were located in completely different branches of the MST, were found in patients admitted to hospital within the same time period. The two stx2dpositive isolates were found in patients admitted to hospital in 2001 and 2003 respectively.

\section{Discussion}

The global burden of disease caused by $E$. coli infections is tremendous. E. coli gastroenteritis alone is responsible for 800,000 deaths annually [21]. Vaccine development aiming at the most common enteropathogenic E. coli, the enterotoxigenic E. coli (ETEC), is therefore of imperative importance. Experimental studies of a second generation ETEC vaccine are promising [22]. E. coli causes the majority of urinary tract infections [23], a considerable part of blood stream infections [2], and is a commonly found bacterial species in infections of the central nervous system of newborns and patients with anatomic or surgical defects [24]. The amount of suffering, as well as the threat of antimicrobial resistance, has brought about efforts to develop a vaccine and alternative treatment options, also for extraintestinal infections caused by $E$. coli. The design of such strategies is challenging, among other factors because E. coli strains carrying ExPEC characteristics are prevalent in the feces of healthy individuals $[25,26]$. Complicating the matter further, advanced age may influence "normality" of fecal flora. A recent study describes the increased diversity and virulence in content of fecal E. coli in the elderly as compared to young adults [27], suggesting the possibility that $E$. coli in extraintestinal infections in the elderly contain more virulence factors than in younger patients. On the other hand, since advanced age is associated with immune remodeling, resulting in increased susceptibility to infectious disease [28] and since E. coli in extraintestinal infections in liver transplant patients are shown to carry less virulent strains [29], the opposite may also be possible. The E. coli virulence load in skin and soft tissue infections is not agedependent [30], but there are few age-focused studies describing bacteremia strains. With a growing population of elderly and very old 
[31], future development of ExPEC vaccine or non-antibiotic treatment options should be aimed at bacterial properties also relevant for extraintestinal E. coli infections in advanced age.

In the current study, clinical data from 212 patients with communityacquired bacteremia caused by $E$. coli were analyzed and compared to the characteristics of the corresponding $E$. coli isolates with special emphasis on the following factors: age, age-related phenomena and severity of infection. Using MLVA, we found a high degree of molecular diversity among the strains, and one MTC was associated with having $\geq 1$ comorbid illness and $\geq 1$ "atypical symptom"; another was associated with leukopenia, whereas a third MTC was associated with urinary tract origin and acted to prevent against gastrointestinal origin. Neither antimicrobial resistance nor MTC were associated with severity of infection. Using traditional phylogenetic analyses, similar results have been reported, that is, some associations between $E$. coli characteristics and certain clinical and demographic variables, but no association with severity of infection. A Danish study of $196 \mathrm{E}$. coli bacteremia isolates with a urinary tract origin found that certain virulence-associated genes were associated with gender and with whether the infection was acquired outside or inside hospital, as well as to different phylogenetic groups, but no relation was found between bacterial factors and mortality [9]. An Irish study reported that bacteremia isolates acquired outside hospital had a higher virulence factor score than nosocomial strains. However, this study did not look into patient outcomes [32]. A French study of the genetics of 161 consecutive E. coli bacteremia isolates showed that bacteremia strains are distributed over the entire span of $E$. coli phylogenetic diversity and that clonal complex, in spite of not being associated with severe sepsis or unfavorable outcomes represent important direction for future research in pathogenesis and comparative genomics [33]. A selection of 60 of the 161 strains was used to induce sepsis in genetically identical mice, and the authors found strong associations between phylogenetic group, virulence factor content and observed virulence, but this did not translate into severity of infection in the patients from whom the isolates originated from in the first place. The results indicate that blood stream infection is dependent on many, often heterogeneous, host factors (for instance individual immune status), or that virulence factors effectively killing mice may not be determinants of severity in human infection [34].

Given that the grouping of ECOR strains of main phylogenetic group A shown in Figure 2a indicates a phylogenetic relationship between group A ECOR strains and the bacteremia isolates within this MST branch, less than 3\% of isolates of urinary tract origin represented phylogroup A. The statistical power in this evaluation was low, and may not be comparable to a level of $15 \%$ of isolates found in a study of $E$. coli bacteremia of urinary tract origin [35]. Our findings support the insight that also phylogroup A E. coli can cause severe infection from a variety of sources, including urinary tract origin.

The grouping of stx2-positive isolates in the MST, of which five out of six were subtype stx $2 c$ and found in patients admitted to hospital during the same time period, as were the two other patients diseased by $s t x 2 c$-positive isolates, indicated an unnoticed outbreak of $s t \times 2 c$ carrying bacteriophage, infecting E. coli of different genotypes. However, as previously reported, st $\times 2$-status of the isolates was associated neither to age nor outcome [19].

E. coli is a bacterial species of great genomic variation. Whole Genome Sequencing (WGS) has shown that only about $20 \%$ of E. coli genes are part of the core genome of this species [36]. There is no direct relationship between $E$. coli pathotype and phylogenetic lineage, and an unambiguous distinction of ExPEC and commensals is complicated, as strains with ability to cause extraintestinal infection are facultative pathogens and belong to the normal flora of many healthy individuals [37]. Moreover, there is a notion of extensive horizontal gene transfer and the transmission of the genetic source of whole pathotypes in a single step via mobile elements [37].

The MLVA molecular-epidemiological typing tool is gaining popularity because it is simple, cheap and fast [14]. It is a high resolution molecular tool primarily developed for outbreak detection and epidemiological surveillance [15], and generally considered less appropriate for describing phylogeny. MLVA is based on the repetitive DNA-sequences, known as Variable Number of Tandem Repeats (VNTRs), which are selected on the basis of their high degree of variation in number of repeats for each locus. Thus, by using MLVA the genome is characterized in a completely different way than through Multi-Locus Enzyme Electrophoresis (MLEE) and Multi-Locus Sequence Typing (MLST), which evaluate the presence of core enzymes and housekeeping genes, respectively. However, VNTRs may be part of inheritance mechanisms and evolution in a much broader perspective than the inheritance of absolutely necessary abilities. Tandem repeats located within gene-coding regions are frequent in the human genome as well as in other organisms studied, and is associated with neurodegenerative diseases [38] and with the imprint of childhood adversities on the genetic risk factors for adult depression [39]. We therefore speculate that VNTRs may be involved in the inheritance of biological vulnerability, thereby justifying this explorative study of host and bacterial determinants of severe infection by MLVA. We are, however, aware that the methodological uncertainties may be regarded as a weakness of our study. It has already been proven that the original 7-locus version of the MLVA-assay used in this study is able to type all ECOR strains, and as expected, does not assign them to the main phylogenetic groups defined by MLEE [17]. However, the recently updated version was found to type multidrug resistant $E$. coli lineages [40], and MLVA has been used for phylotyping STEC O157 [41]. MLVA has a methodological drawback when the VNTR is present within a coding region or promoter and can thus be too discriminatory [42]. In the MLVA-assay used in this study, two of the VNTRs are located in gen-coding areas [17]. Similar studies have been performed, focusing on phylogeny and virulence factors, however, without concentrating on patient age, and with negative results regarding associations with outcome. MLVA explores the bacterial genome from a totally different angle, and we wanted to explore if a generic $E$. coli MLVA protocol could differentiate $E$. coli strains into groups associated with age and outcome.

The major strengths of this study are the relatively high number of strains and patients included, and the consecutive sampling over a five-year period. When considering antimicrobial resistance and associations between MTCs with low number of strains, the statistical power may be too low to detect significant microbial differences.

\section{Conclusions}

It is not known if severely infected elderly are diseased by different groups of $E$. coli than younger patients. In the current study of community-acquired E. coli bacteremia isolates we focused on patient age and age-associated clinical presentation as well as infection outcome. Our study revealed great diversity among the strains, and none of the genotypes as assessed by MLVA was associated with outcome. Although no genotype was associated with patient age, one of them was associated with the age-related clinical phenomena of having $\geq 1$ comorbid illness and a clinical presentation with $\geq 1$ "atypical symptom" of infection. This indicated that further study of the bacterial population found in the elderly with ExPEC infections is highly relevant, with a view to 
Citation: Wester AL, Melby KK, Wyller TB, Dahle UR (2014) E. coli Bacteremia Strains - High diversity and Associations with Age-related Clinical Phenomena. Clin Microbial 3: 140. doi:10.4172/2327-5073.1000140

designing ExPEC vaccine or novel ExPEC treatment regimens, which will also be effective in advanced age.

\section{Ethical Considerations}

The study was approved by the Regional Committee for Ethics in Medical Research and by the Norwegian Data Inspectorate, which have given permission to carry out the study without the patients' consent.

\section{Funding}

The study was performed without specific funding.

\section{Authors' Contribution}

ALW participated in the concept and design of the study, gathered data on bacteremia, serum markers of infection, clinical data on presentation of infection, performed the analyses and participated in the writing of the manuscript.

KKM participated in the design and the writing of the manuscript

TBW participated in the design, the data interpretation and the writing of the manuscript.

URD participated in the design, the data interpretation and the writing of the manuscript

\section{Acknowledgements}

Trine-Lise Stavnes and Inger Løbersli are acknowledged for excellen technical assistance.

\section{References}

1. Diekema DJ, Pfaller MA, Jones RN, Group SP (2002) Age-related trends in pathogen frequency and antimicrobial susceptibility of bloodstream isolates in North America: SENTRY Antimicrobial Surveillance Program, 1997-2000. International journal of antimicrobial agents 20: 412-418.

2. Fluit AC, Jones ME, Schmitz FJ, Acar J, Gupta R, et al. (2000) Antimicrobial susceptibility and frequency of occurrence of clinical blood isolates in Europe from the SENTRY antimicrobial surveillance program, 1997 and 1998. Clinical infectious diseases of America 30: 454-460.

3. Laupland KB, Gregson DB, Church DL, Ross T, Pitout JD (2008) Incidence, risk factors and outcomes of Escherichia coli bloodstream infections in a large Canadian region. Clinical microbiology and infection 14: 1041-1047.

4. Peralta G, Sánchez MB, Garrido JC, De Benito I, Cano ME, et al. (2007) Impact of antibiotic resistance and of adequate empirical antibiotic treatment in the prognosis of patients with Escherichia coli bacteraemia. J Antimicrob Chemother 60: 855-863.

5. Johnson JR, Russo TA (2002) Extraintestinal pathogenic Escherichia coli: "the other bad E coli". J Lab Clin Med 139: 155-162.

6. Picard B, Garcia JS, Gouriou S, Duriez P, Brahimi N, et al. (1999) The link between phylogeny and virulence in Escherichia coli extraintestinal infection. Infect Immun 67: 546-553.

7. Jaureguy F, Carbonnelle E, Bonacorsi S, Clec'h C, Casassus P, et al. (2007) Host and bacterial determinants of initial severity and outcome of Escherichia coli sepsis. Clinical microbiology and infection 13: 854-862.

8. Lefort A, Panhard X, Clermont O, Woerther PL, Branger C, et al. (2011) Host factors and portal of entry outweigh bacterial determinants to predict the severity of Escherichia coli bacteremia. J Clin Microbiol 49: 777-783.

9. Skjøt-Rasmussen L, Ejrnæs K, Lundgren B, Hammerum AM, Frimodt-Møller N (2012) Virulence factors and phylogenetic grouping of Escherichia coli isolates from patients with bacteraemia of urinary tract origin relate to sex and hospitalvs. community-acquired origin. Int J Med Microbiol 302: 129-134.

10. Opal SM, Girard TD, Ely EW (2005) The immunopathogenesis of sepsis in elderly patients. Clin Infect Dis 41: S504-512.

11. De Gaudio AR, Rinaldi S, Chelazzi C, Borracci T (2009) Pathophysiology of sepsis in the elderly: clinical impact and therapeutic considerations. Curr Drug Targets 10: 60-70.

12. Dobrindt U, Hacker J (2008) Targeting virulence traits: potential strategies to combat extraintestinal pathogenic E. coli infections. Curr Opin Microbiol 11 409-413.

13. Russo TA, Beanan JM, Olson R, Genagon SA, MacDonald U, et al. (2007) A killed, genetically engineered derivative of a wild-type extraintestinal pathogenic E. coli strain is a vaccine candidate. Vaccine 25: 3859-3870.

14. Sabat AJ, Budimir A, Nashev D, Sa-Leao R, van Dijl J, et al. (2013) Overview of molecular typing methods for outbreak detection and epidemiological surveillance. Euro surveillance : bulletin Europeen sur les maladies transmissibles $=$ European communicable disease bulletin .

15. Lindstedt BA, Torpdahl M, Vergnaud G, Le Hello S, Weill FX, et al. (2013) Use of multilocus variable-number tandem repeat analysis (MLVA) in eight European countries, 2012. Euro surveillance : bulletin Europeen sur les maladies transmissibles = European communicable disease bulletin.

16. Løbersli I, Haugum K, Lindstedt BA (2012) Rapid and high resolution genotyping of all Escherichia coli serotypes using 10 genomic repeat-containing loci. J Microbiol Methods 88: 134-139.

17. Lindstedt BA, Brandal LT, Aas L, Vardund T, Kapperud G (2007) Study of polymorphic variable-number of tandem repeats loci in the ECOR collection and in a set of pathogenic Escherichia coli and Shigella isolates for use in a genotyping assay. J Microbiol Methods 69: 197-205.

18. Wester AL, Dunlop O, Melby KK, Dahle UR, Wyller TB (2013) Age-related differences in symptoms, diagnosis and prognosis of bacteremia. BMC Infect Dis 13: 346 .

19. Wester AL, Brandal LT, Dahle UR (2013) Extraintestinal pathogenic Escherichia coli carrying the Shiga Toxin gene stx2. J Clin Microbiol 51: 4279-4280.

20. Magiorakos AP, Srinivasan A, Carey RB, Carmeli Y, Falagas ME, et al. (2012) Multidrug-resistant, extensively drug-resistant and pandrug-resistant bacteria: an international expert proposal for interim standard definitions for acquired resistance. Clinical microbiology and infection 18: 268-281.

21. Liu L, Johnson HL, Cousens S, Perin J, Scott S, et al. (2012) Global, regional and national causes of child mortality: an updated systematic analysis for 2010 with time trends since 2000. Lancet 379: 2151-2161.

22. Holmgren J, Bourgeois L, Carlin N, Clements J, Gustafsson B, et al. (2013) Development and preclinical evaluation of safety and immunogenicity of an oral ETEC vaccine containing inactivated $E$. coli bacteria overexpressing colonization factors CFA/I, CS3, CS5 and CS6 combined with a hybrid LT/CT B subunit antigen, administered alone and together with $\mathrm{dmLT}$ adjuvant. Vaccine 31: $2457-2464$.

23. Moreno E, Andreu A, Pigrau C, Kuskowski MA, Johnson JR, et al. (2008) Relationship between Escherichia coli strains causing acute cystitis in women and the fecal E. coli population of the host. J Clin Microbiol 46: 2529-2534.

24. Bleck TP (2013) Bacterial meningitis and other nonviral infections of the nervous system. Crit Care Clin 29: 975-987.

25. Escobar-Páramo P, Grenet K, Le Menac'h A, Rode L, Salgado E, et al. (2004) Large-scale population structure of human commensal Escherichia coli isolates. Appl Environ Microbiol 70: 5698-5700.

26. Zhang L, Foxman B, Marrs C (2002) Both urinary and rectal Escherichia col isolates are dominated by strains of phylogenetic group B2. J Clin Microbiol 40: 3951-3955.

27. Vollmerhausen TL, Ramos NL, Gündogdu A, Robinson W, Brauner A, et al. (2011) Population structure and uropathogenic virulence-associated genes of faecal Escherichia coli from healthy young and elderly adults. J Med Microbiol 60: 574-581.

28. Vollmerhausen TL, Ramos NL, Gündogdu A, Robinson W, Brauner A, et al (2011) Population structure and uropathogenic virulence-associated genes of faecal Escherichia coli from healthy young and elderly adults. J Med Microbiol 60: 574-581.

29. Dorrington MG, Bowdish DM (2013) Immunosenescence and novel vaccination strategies for the elderly. Front Immunol 4: 171.

30. Bert F, Huynh B, Dondero F, Johnson JR, Paugam-Burtz C, et al. (2011) Molecular epidemiology of Escherichia coli bacteremia in liver transplant recipients. Transplant infectious disease 13: 359-365.

31. Petkovsek Z, Elersic K, Gubina M, Zgur-Bertok D, Starcic Erjavec M (2009) Virulence potential of Escherichia coli isolates from skin and soft tissue infections. J Clin Microbiol 47: 1811-1817.

32. Cooke NM, Smith SG, Kelleher M, Rogers TR (2010) Major differences exist in 
Citation: Wester AL, Melby KK, Wyller TB, Dahle UR (2014) E. coli Bacteremia Strains - High diversity and Associations with Age-related Clinical Phenomena. Clin Microbial 3: 140. doi:10.4172/2327-5073.1000140

frequencies of virulence factors and multidrug resistance between community and nosocomial Escherichia coli bloodstream isolates. J Clin Microbiol 48 1099-1104.

33. Jaureguy F, Landraud L, Passet V, Diancourt L, Frapy E, et al. (2008) Phylogenetic and genomic diversity of human bacteremic Escherichia coli strains. BMC Genomics 9: 560 .

34. Landraud L, Jaureguy F, Frapy E, Guigon G, Gouriou S, et al. (2013) Severity of Escherichia coli bacteraemia is independent of the intrinsic virulence of the strains assessed in a mouse model. Clinical microbiology and infection 19: 8590.

35. Skjot-Rasmussen L, Olsen SS, Jakobsen L, Ejrnaes K, Scheutz F, et al. (2013) Escherichia coli clonal group A causing bacteraemia of urinary tract origin Clinical microbiology and infection 19: 656-661.

36. Lukjancenko O, Wassenaar TM, Ussery DW (2010) Comparison of 61 sequenced Escherichia coli genomes. Microb Ecol 60: 708-720.

37. Leimbach A, Hacker J, Dobrindt U (2013) E. coli as an all-rounder: the thin line between commensalism and pathogenicity. Curr Top Microbiol Immunol 358: 3-32.
38. Gemayel R, Vinces MD, Legendre M, Verstrepen KJ (2010) Variable tandem repeats accelerate evolution of coding and regulatory sequences. Annu Rev Genet 44: 445-477.

39. Melas PA, Wei Y, Wong CC, Sjoholm LK, Aberg E, et al. (2013) Genetic and epigenetic associations of MAOA and NR3C1 with depression and childhood adversities. The international journal of neuropsychopharmacology 16: 15131528.

40. Naseer U, Olsson-Liljequist BE, Woodford N, Dhanji H, Cantón R, et al. (2012) Multi-locus variable number of tandem repeat analysis for rapid and accurate typing of virulent multidrug resistant Escherichia coli clones. PLoS One 7: e41232.

41. Jenke C, Harmsen D, Weniger T, Rothganger J, Hyytia-Trees E, et al. (2010) Phylogenetic analysis of enterohemorrhagic Escherichia coli O157, Germany, 1987-2008. Emerg Infect Dis 16: 610-616.

42. Lindstedt BA (2005) Multiple-locus variable number tandem repeats analysis for genetic fingerprinting of pathogenic bacteria. Electrophoresis 26: 2567-2582. 